\title{
Pretreatment plasma levels and diagnostic utility of hematopoietic cytokines in cervical cancer or cervical intraepithelial neoplasia patients
}

\author{
Sławomir Lawicki' ${ }^{1}$, Grażyna E. Będkowska ${ }^{1}$, Ewa Gacuta-Szumarska², \\ Paweł Knapp ${ }^{3}$, Maciej Szmitkowski ${ }^{1}$ \\ ${ }^{1}$ Department of Biochemical Diagnostics, Medical University of Bialystok, Poland \\ ${ }^{2}$ Department of Perinatology, Medical University of Bialystok, Poland \\ ${ }^{3}$ Department of Gynecology, Medical University of Bialystok, Poland
}

\begin{abstract}
In this study, we compared plasma levels and the diagnostic utility of hematopoietic growth factors (HGFs) with SCC-Ag in cervical cancer patients in relation to control groups and cervical intraepithelial neoplasia (CIN) patients and healthy subjects. Pretreatment plasma levels of HGFs (SCF, GM-CSF, G-CSF and $\mathrm{M}$-CSF) were determined by the use of immunoenzyme assay (ELISA), and SCC-Ag by chemiluminescent microparticle immunoassay (CMIA). Significantly different concentrations of GM-CSF, G-CSF and M-CSF were observed in the group of patients with cervical cancer and CIN compared to the healthy controls. Significant differences in plasma levels of GM-CSF and M-CSF between cervical cancer and benign lesions patients were also found. The HGFs and SCC-Ag diagnostic specificities received high values. The diagnostic sensitivity and the predictive value of a positive and negative test result were higher for M-CSF than for antigen SCC in the cancer group. The M-CSF area under the ROC curve (AUC) was the largest from hematopoietic cytokines and SCC-Ag. These results suggest the potential utility of M-CSF as a good candidate for a marker of cervical cancer as well as benign lesions of this organ (CIN). (Folia Histochemica et Cytobiologica 2012, Vol. 50, No. 2, 213-219)
\end{abstract}

Key words: cervical cancer, HGFs, SCC-Ag, tumor markers

\section{Introduction}

Cervical cancer is a leading cause of morbidity and mortality among women worldwide, especially in developing countries [1]. Findings from clinical and epidemiologic research convincingly support human papillomavirus (HPV) as the major risk factor for carcinoma of the cervix [2]. Promiscuity, smoking history, and low socio-economic status have also been established as risk factors for this malignancy [3].

The Bethesda System (TBS), which was formulated to standardize the reporting of the Papanico-

Correspondence address: M. Szmitkowski, Department of Biochemical Diagnostics, Medical University of Bialystok,

Waszyngtona 15a, 15-269 Bialystok, Poland; tel.: + 48857468587 , fax: + 48857468585 ; e-mail: zdb@umwb.edu.pl laou test (known as Pap smear) results, is the most important diagnostic technique for detecting pre-invasive cervical cancer [3]. Some antigens have also been measured in the sera of patients with this gynecological cancer and have been related to the clinical course of disease [4].

Stem cell factor (SCF), granulocyte-macrophagecolony stimulating factor (GM-CSF), granulocyte-colony stimulating factor (G-CSF) and macrophage-colony stimulating factor (M-CSF) also known as colony stimulating factor-1 (CSF-1) are members of the group of cytokines called hematopoietic growth factors (HGFs). Their biological role is to regulate the proliferation, mobility, survival and differentiation of hematopoietic progenitor cells [5]. Moreover, many human non-hematopoietic tumors have been shown to express increased levels of HGFs. Furthermore, these cytokines play a role in the pathogenesis of cancer disease [6-8]. 
M-CSF, its receptor (M-CSFR) and c-fms (protooncogene encodes a transmembrane glycoprotein which is identical to the receptor for M-CSF) overexpressions were confirmed on the lines of cervical cancer in comparison with the patients with benign lesions (CIN) and to the control group. The blockade of M-CSFR resulted in the inhibition of tumor growth and the intensification of the apoptosis processes in cancer cells $[9,10]$. The production of G-CSF and GM-CSF has been demonstrated in a number of nonhematologic malignancies including tumors of the cervix [11-13]. Tissue expression of high levels of G-CSF by tumor cells, or elevated levels of G-CSF in patient serum samples, have been found to be associated with an aggressive malignant nature of the tumor and poor patient outcomes [14]. The most popular theory which explains the aggressive nature of G-CSF-associated cancers is the autocrine stimulation of tumor growth by tumor cells which secrete G-CSF and carry its receptor on their surface $[12,14]$. The presence of receptors for SCF has also been confirmed on the lines of ovarian and cervical cancer cells $[8,15]$.

Cervical intraepithelial neoplasia (CIN) is a morphologically defined lesion associated with the development of cervical carcinoma [16]. There is still little knowledge concerning plasma levels of hematopoietic cytokines in subjects with benign lesions of this organ.

The aim of this study was to determine the plasma level and the diagnostic utility of selected HGFs (SCF, GM-CSF, G-CSF, M-CSF) in comparison with the commonly accepted tumor marker (SCC-Ag) in cervical cancer patients in relation to the control groups, cervical intraepithelial neoplasia patients, and healthy controls. Additionally, the diagnostic criteria and receiver-operating characteristics curve (ROC) for the tested cytokines and SCC-Ag were defined.

\section{Material and methods}

Patients. The study consisted of 50 patients with squamous cell carcinoma (SCC) of the cervix (women aged 25-77; years $\mathrm{SD}=12.70$ ) diagnosed by the Gynecology group. The control groups comprised 30 cervical intraepithelial neoplasia (CIN) patients (women aged 23-75; years SD $=13.65$ ) and 45 healthy volunteers (women aged 23-72 years; years $\mathrm{SD}=12.74)$. The cervical cancer patients and the control group (benign lesions) were treated in the Department of Gynecology, Medical University Hospital in Białystok, Poland between 2005 and 2009. None of the patients had received chemoor radiotherapy before blood sample collection.

The pretreatment staging procedures included: a cytological examination according to the TBS system, physical
Table 1. Characteristics of cervical cancer patients and control groups: cervical intraepithelial neoplasia (CIN) patients and healthy subjects

\begin{tabular}{|llc|}
\hline Study group & & $\begin{array}{c}\text { Number } \\
\text { of patients }\end{array}$ \\
\hline $\begin{array}{l}\text { Cervical cancer patients } \\
\text { (type squamous cell carcinoma) }\end{array}$ & 50 \\
\hline Median age (range) & & $49(25-77)$ \\
\hline Tumor stage & 0 (CIN III) & 9 \\
& IA & 12 \\
& IB & 14 \\
& IIA & 8 \\
& IIB & 7 \\
\hline Depth of tumor invasion & $\mathrm{T}_{\text {is }}$ & 9 \\
(T factor) & $\mathrm{T}_{1}$ & 26 \\
& $\mathrm{~T}_{2}$ & 15 \\
\hline Nodal metastases & $\mathrm{N}_{0}$ & 50 \\
(N factor) & & 50 \\
\hline Distant metastases & $\mathrm{M}_{0}$ & \\
(M factor) & & 30 \\
\hline Cervical intraepithelial neoplasia & & 45 \\
Median age (range) & & 16 \\
Numbers: & & \\
CIN I & & \\
CIN II & & \\
\hline Healthy subjects & & \\
\hline Median age (range) & & \\
\hline
\end{tabular}

and blood examinations and ultrasound scanning. Cervical cancer and CIN histopathology was established in all cases. The healthy women group also underwent a gynecological, including a cytological, examination according to the TBS system prior to blood collection. In addition, a reproductive organ ultrasound scan was performed where necessary. Table 1 shows the tested groups. The clinical stages and histological classification based on the criteria of the International Federation of Gynecology and Obstetrics (FIGO) and the World Health Organization (WHO) were as follows: 35 patients in stage I, and 15 in stage II of the disease.

The study was approved by the local Ethics Committee (numbers: R-I-003/408/2004; R-I-002/262/2010) and all the patients gave informed consent for their participation in the study.

Biochemical analyses. Venous blood samples were collected from each patient into a heparin sodium tube, centrifuged at 1,000 rpm for $15 \mathrm{~min}$. to obtain plasma samples, and stored at $-85^{\circ} \mathrm{C}$ until assayed. Hematopoietic cytokines were measured with enzyme-linked immunosorbent assay (ELISA) (Quantikine Human HGFs Immunoassay; R\&D Systems, Abingdon, UK), according to the manufacturer's protocols. The intra-assay coefficient of variation (CV\%) 
Table 2. Plasma levels of hematopoietic cytokines and SCC-Ag in cervical cancer patients and in control groups: cervical intraepithelial neoplasia patients (CIN) and healthy subjects

\begin{tabular}{|c|c|c|c|}
\hline & Cervical cancer & Cervical intraepithelial neoplasia & Healthy subjects \\
\hline $\begin{array}{l}\text { SCF }[\mathbf{p g} / \mathbf{m l}] \\
\text { Median } \\
\text { Range } \\
\text { p }\end{array}$ & $\begin{array}{c}713.94 \\
384.81-1,136.55 \\
\text { NS }\end{array}$ & $\begin{array}{c}817.40 \\
479.65-922.53 \\
\text { NS }\end{array}$ & $\begin{array}{c}687.67 \\
398.60-1,020.82\end{array}$ \\
\hline $\begin{array}{l}\text { GM-CSF }[\mathbf{p g} / \mathbf{m l}] \\
\text { Median } \\
\text { Range } \\
\text { p }\end{array}$ & $\begin{array}{c}0.23^{* / * *} \\
0.03-4.45 \\
<0.001 /=0.004\end{array}$ & $\begin{array}{c}0.44^{*} \\
0.03-3.14 \\
=0.001\end{array}$ & $\begin{array}{c}0.15 \\
0.03-2.35\end{array}$ \\
\hline $\begin{array}{l}\text { G-CSF }[\mathbf{p g} / \mathbf{m l}] \\
\text { Median } \\
\text { Range } \\
\text { p }\end{array}$ & $\begin{array}{c}20.05^{*} \\
0.11-64.70 \\
<0.001\end{array}$ & $\begin{array}{c}19.60^{*} \\
10.42-46.43 \\
<0.001\end{array}$ & $\begin{array}{c}13.31 \\
3.11-29.55\end{array}$ \\
\hline $\begin{array}{l}\text { M-CSF }[\mathbf{p g} / \mathbf{m l}] \\
\text { Median } \\
\text { Range } \\
\text { p }\end{array}$ & $\begin{array}{c}500.45^{* * * *} \\
311.95-2,513.75 \\
<0.001 /=0.003\end{array}$ & $\begin{array}{c}415.30^{*} \\
102.15-597.60 \\
=0.024\end{array}$ & $\begin{array}{c}290.82 \\
119.62-455.60\end{array}$ \\
\hline $\begin{array}{l}\text { SCC-Ag [ng/ml] } \\
\text { Median } \\
\text { Range } \\
\text { p }\end{array}$ & $\begin{array}{c}1.10^{*} \\
0.50-159.00 \\
=0.040\end{array}$ & $\begin{array}{c}1.00 \\
0.50-1.60 \\
\mathrm{NS}\end{array}$ & $\begin{array}{c}0.90 \\
0.60-1.80\end{array}$ \\
\hline
\end{tabular}

*Statistically significant compared to healthy subject group; **statistically significant compared to benign cervical lesions group; NS - not significant

of SCF is reported by the manufacturers to be $2.0 \%$ at a mean concentration of $655 \mathrm{pg} / \mathrm{ml}, \mathrm{SD}=12.9$, of GM-CSF - to be $9.5 \%$ at a mean concentration of $3.68 \mathrm{pg} / \mathrm{ml}$, $\mathrm{SD}=0.35$, of G-CSF - to be $2.8 \%$ at a mean concentration of $280 \mathrm{pg} / \mathrm{ml}, \mathrm{SD}=7.8$, of M-CSF - to be $3.4 \%$ at a mean concentration of $227 \mathrm{pg} / \mathrm{ml}, \mathrm{SD}=7.7$.

Plasma concentrations of SCC-Ag were measured by chemiluminescent microparticle immunoassay (CMIA) (Abbott, Chicago, IL, USA). The intra-assay CV for SCC-Ag is reported by the manufacturer of the assay kit to be $5.1 \%$ at a mean concentration of $1.42 \mathrm{ng} / \mathrm{ml}, \mathrm{SD}=0.072$.

Statistical analysis. A preliminary statistical analysis ( $\mathrm{c}^{2}$ test) revealed that the distribution of cytokine and tumor marker levels did not follow normal distribution. Consequently, the Mann-Whitney U-test was used for statistical analysis. The Spearman rank correlation was used in the correlation analyses. Data was presented as median and range. Statistically significant differences were defined as comparisons resulting in $\mathrm{p}<0.05$. The cut-off of HGFs (SCF - 969.30 $\mathrm{pg} / \mathrm{ml}$, GM-CSF $-1.38 \mathrm{pg} / \mathrm{ml}$, G-CSF $-25.05 \mathrm{pg} / \mathrm{ml}$ and $\mathrm{M}-\mathrm{CSF}-406.85 \mathrm{pg} / \mathrm{ml})$ and SCC-Ag $(1.80 \mathrm{ng} / \mathrm{ml})$ were used at the specificity higher than $95 \%$ (calculated from healthy blood donors). Statistical analyses were conducted using the
STATISTICA 8 PL program (StatSoft Inc., Tulsa, OK, USA). Additionally, the diagnostic criteria, such as sensitivity, specificity, predictive value of a positive (PV-PR) and negative (PV-NR) test result, and the area under the ROC curve (AUC), were determined. The construction of the ROC curves was performed using GraphRoc Program for Windows.

\section{Results}

Table 2 shows the median and range of the investigated HGFs and SCC-Ag in the tested groups. The medians of GM-CSF, G-CSF and M-CSF $(0.23 \mathrm{pg} / \mathrm{ml}$, $20.05 \mathrm{pg} / \mathrm{ml}$ and $500.45 \mathrm{pg} / \mathrm{ml}$ respectively) as well as SCC-Ag $(1.10 \mathrm{ng} / \mathrm{ml})$ in the group with cervical cancer, were statistically significantly different compared to the healthy women group $(0.15 \mathrm{pg} / \mathrm{ml}, 13.31 \mathrm{pg} / \mathrm{ml}$; $290.82 \mathrm{pg} / \mathrm{ml}$ and $0.90 \mathrm{ng} / \mathrm{ml}$ respectively) ( $\mathrm{p}<0.001$ for the above-mentioned cytokines and $\mathrm{p}=0.040$ for antigen SCC respectively).

Similarly, the medians of GM-CSF $(0.44 \mathrm{pg} / \mathrm{ml})$, G-CSF (19.60 pg/ml) and M-CSF (415.30 pg/ml) in the group of CIN patients were statistically significantly higher compared to the healthy subjects ( $\mathrm{p}=$ $=0.001 ; \mathrm{p}<0.001$ and $\mathrm{p}=0.024$, respectively). We 
Table 3. Analysis of the diagnostic parameters of hematopoietic cytokines and SCC-Ag

\begin{tabular}{|l|c|c|c|c|}
\hline & $\begin{array}{c}\text { Diagnostic } \\
\text { sensitivity }\end{array}$ & $\begin{array}{c}\text { Diagnostic } \\
\text { specificity }\end{array}$ & PV-PR & PV-NR \\
\hline SCF & $8 \%$ & $93 \%$ & $57 \%$ & $48 \%$ \\
\hline GM-CSF & $6 \%$ & $93 \%$ & $50 \%$ & $52 \%$ \\
\hline M-CSF & $54 \%$ & $93 \%$ & $90 \%$ & $65 \%$ \\
\hline G-CSF & $16 \%$ & $93 \%$ & $73 \%$ & $50 \%$ \\
\hline SCC-Ag & $26 \%$ & $93 \%$ & $81 \%$ & $53 \%$ \\
\hline
\end{tabular}

also observed statistically significantly different plasma levels of GM-CSF and M-CSF between the groups of patients with cervical cancer and those with cervical intraepithelial neoplasia $(0.23 \mathrm{pg} / \mathrm{ml} ; 0.44 \mathrm{pg} / \mathrm{ml}$ and $500.45 \mathrm{pg} / \mathrm{ml} ; 415.30 \mathrm{pg} / \mathrm{ml}$ respectively) (Table 2).

Table 3 shows the HGFs' diagnostic parameters compared to SCC-Ag. Among all the tested parameters, only M-CSF showed the highest diagnostic sensitivity $(54 \%)$. The combined use of the tested hematopoietic cytokines with antigen SCC resulted in an increase in sensitivity range. A maximum diagnostic sensitivity $(68 \%)$ was obtained for the combination of M-CSF with SCC-Ag (Table 4). The diagnostic specificities of the tested cytokines and SCC-Ag received high equal values (93\%) (Table 3). The M-CSF predictive value of a positive test result - PV-PR (90\%) and predictive value of a negative test result - PV-NR $(65 \%)$ were higher than those of SCC-Ag ( $81 \%$ and $53 \%$ respectively) (Table 3$)$. The combined use of the tested parameters resulted in a decrease in the PV-PR to the range of $70 \%$, and in an increase in the PV-NR to the value of $71 \%$ (Table 4).

The area under the ROC curve indicates the clinical usefulness of a tumor marker. In this study, the M-CSF area (0.729) under the ROC curve (Figure 1) is the largest from all HGFs, and far larger than that of SCC-Ag (0.527). Moreover, only the area under the ROC curve for M-CSF was statistically larger compared to $\mathrm{AUC}=0.5$ (borderline of diagnostic usefulness of the test) $(p<0.001)$ (Figure 1$)$.

Spearman rank correlation was used in the dependence analysis between the investigated parameters. There was no significant positive or negative correlation between hematopoietic cytokines and SCC-Ag concentrations in the cervical cancer or benign cervical lesions groups.

\section{Discussion}

Hematopoietic cytokines participate in hematopoiesis regulation, but they also appear to play a crucial role in the development of cancers of different loca-
Table 4. Combined analysis of the diagnostic parameters of hematopoietic cytokines with SCC-Ag

\begin{tabular}{|l|c|c|c|}
\hline & $\begin{array}{c}\text { Diagnostic } \\
\text { sensitivity }\end{array}$ & PV-PR & PV-NR \\
\hline SCF + SCC-Ag & $32 \%$ & $73 \%$ & $53 \%$ \\
\hline GM-CSF + SCC-Ag & $28 \%$ & $70 \%$ & $52 \%$ \\
\hline M-CSF + SCC-Ag & $68 \%$ & $85 \%$ & $71 \%$ \\
\hline G-CSF + SCC-Ag & $36 \%$ & $75 \%$ & $55 \%$ \\
\hline
\end{tabular}

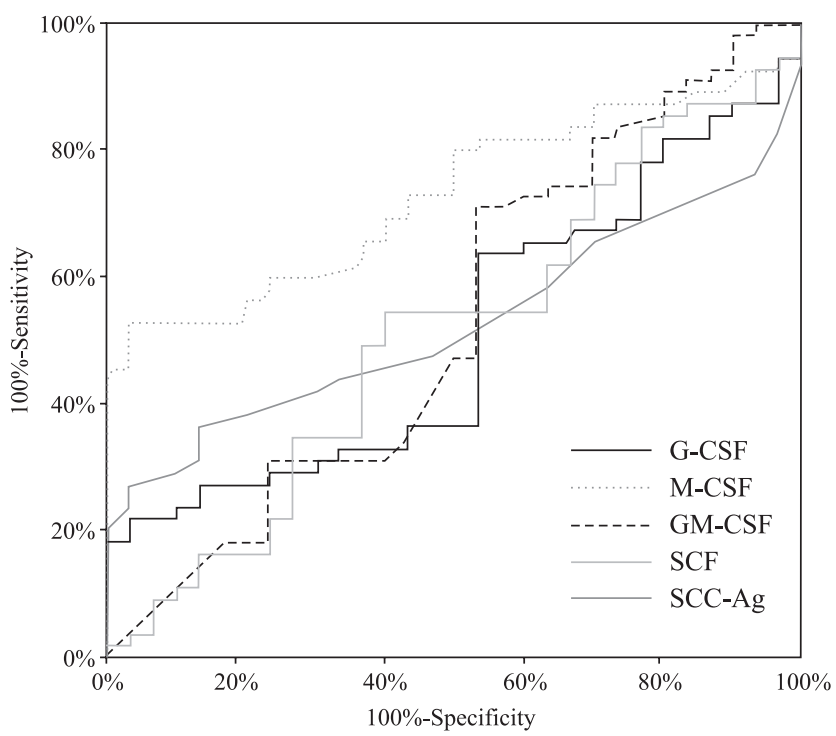

Figure 1. ROC curves for $\mathrm{SCF}(\mathrm{AUC}=0.511 ; \mathrm{NS})$, GM-CSF $(\mathrm{AUC}=0.529 ; \mathrm{NS}), \mathrm{G}-\mathrm{CSF}(\mathrm{AUC}=0.507 ; \mathrm{NS})$, M-CSF $($ AUC $=0.729 ; \mathrm{p}<0.001)$ and SCC-Ag $(\mathrm{AUC}=0.527 ; \mathrm{NS})$ in cervical cancer patients

tions [17-20]. Elevated levels of HGFs have also been demonstrated in the sera of patients with malignancies of the reproductive organ, e.g. increased levels of M-CSF in ovarian [21] or endometrial [22], and G-CSF in cervical $[11,13]$ cancer patients.

In this study, the plasma levels and the diagnostic utility of selected hematopoietic cytokines were investigated. In the group of cervical cancer patients, the plasma levels of GM-CSF, G-CSF and M-CSF were statistically significantly different compared to the healthy controls. These markers have been found to be capable of discriminating cervical cancer patients from those without malignant transformation. Comparable levels of these three cytokines (but without statistical significance), were observed in the study of Punnonen et al. [23], although a different type of research material was used and the tested group of cervical cancer patients was much smaller $(n=23)$. Significant data for G-CSF has also been observed in 
the studies of other authors who additionally confirmed auto - and/or paracrine growth stimulation of tumor cells by this cytokine in different histological types of cervical cancer: carcinosarcoma [24], small-cell [14] and squamous cell [11-13] carcinoma. The tissue expression of high levels of G-CSF by tumor cells or elevated levels of G-CSF in patient serum samples have been found to be associated with an aggressive malignant nature of the tumor and poor patient outcomes [12,14].

In the present study, which is a continuation of our earlier investigation, a higher level of M-CSF was also observed [25]. Furthermore, the key role of this factor and its receptor in the initiation of carcinogenesis and tumor growth stimulation has been suggested in previous publications by other authors $[9,10]$.

The plasma concentrations of SCC-Ag were significantly higher in the cervical cancer group compared to the healthy controls. Our test data is in agreement with the results of other researchers regarding the diagnostic usefulness of antigen SCC in this malignancy [26-28]. Moreover, its prognostic significance, both for recurrence-free and overall survival, has been confirmed by other researchers in the early stages of cervical cancer [29].

The distribution of SCF in the healthy donors and in the cervical cancer patients was not significantly different. These research results are consistent with the findings from our previous study [25], although Inoue et al. [8] and Ohwada et al. [15] observed overexpression of mRNA for SCF and its receptor (c-kit) in cervical cancer patients. Recently, the participation of this receptor in cervical carcinogenesis associated with the presence of human papillomavirus (HPV) has been indicated [30]. Contradicting the results published in these papers, Wang et al. [31] observed low expression of c-kit, though in a very rare histological type of this malignancy - small cell carcinoma.

In the group of CIN patients, the plasma levels of GM-CSF, G-CSF and M-CSF were statistically significantly higher compared to the healthy subjects. Only these three cytokines were found to be capable of discriminating patients from these two control groups. Previously, other authors have found significantly higher concentrations of M-CSF in patients who either tested positive for human papillomavirus or presented with benign lesions of the cervix. Rony et al. [32] suggested that HPV oncogenes are not only responsible for the immortalization of cervical epithelial cells, but may also be responsible for the activation of M-CSF in CIN, which leads to the concomitant establishment of an autocrine loop. The inflammatory process and granulocytosis may be an explanation for the higher levels of G-CSF both in benign and malignant lesions of the cervix [13]. The investigations of Zijlmans et al. [33] and Czygier et al. [34] are in agreement with our data, and support the hypothesis that GM-CSF (among other cytokines) induces an inflammatory response which assists in tumor growth and progression.

Our results revealed statistically considerably lower levels of GM-CSF in the group of cervical cancer compared to the benign cervical lesions patients. Some authors have observed a down-regulation of the expression level of proinflammatory cytokines such as GM-CSF in cervical cancer, but only in in vitro conditions [35]. Interestingly, in our study M-CSF levels in the cancer group were significantly higher compared to CIN patients. Our results confirm the findings of other researchers $[9,32]$. On the other hand, this data contradicts that of Punnonen et al. [23] who postulated that malignant and benign tumors generally induce similar levels of cytokine production. However, these authors compared (among other gynecological malignancies) cervical cancer patients with benign ovarian and uterine lesions patients only, and purely in peritoneal fluids [23].

The ideal tumor marker should have a high sensitivity and a high specificity in order to discriminate cancer patients from healthy subjects, or from patients with benign conditions, and should be secreted into circulation in concentrations proportional to tumor burden and activity. It should also have high predictive values and should correlate with the tumor stage. The criteria for $100 \%$ specificity and $100 \%$ sensitivity have to date not been fulfilled by any of the known tumor markers. In the current study, the M-CSF sensitivity was the highest (54\%) from all the tested parameters, higher even than the sensitivity for SCC-Ag (26\%).

The results of our study indicate that M-CSF can be a better diagnostic tool than SCC-Ag in the early diagnosis of cervical cancer. This confirms the results obtained from our previous study concerning M-CSF, although the tested group was only half as large [25].

The combined use of the tested cytokines resulted in an increase in the sensitivity to the maximum range $(68 \%)$ for the combination of M-CSF with SCC-Ag. These results are very similar to those obtained by us in previous studies, as well as to those of other authors but in reference to cancers of various locations, for example ovarian carcinoma $[21,36]$ or pancreatic cancer [18].

The HGFs and SCC-Ag diagnostic specificities were high $-93 \%$. This is in agreement with our previous studies on this malignancy [25], and in the course of breast [37] or ovarian cancer [36].

In the present investigation, M-CSF proved to have higher PV-PR and PV-NR values than SCC-Ag (90\%; 
$65 \%$ and $81 \% ; 53 \%$ respectively). Previously, we obtained similar predictive values of hematopoietic cytokines and SCC-Ag in this type of malignancy $(83 \% ; 69.7 \%$ and $72.7 \% ; 51 \%$ respectively), although a cervical cancer group consisted only of 25 patients [25]. The combined use of the tested parameters resulted in a decrease in the PV-PR and in an increase of the PV-NR to the range of $71 \%$. The results of the current study support our previous findings concerning breast cancer [37].

The area under the ROC curve indicates the diagnostic power of a tumor marker. In the present study, the ROC area of M-CSF was larger than the SCC-Ag ROC area $(\mathrm{AUC}=0.729$ and $\mathrm{AUC}=0.527$ respectively). Additionally, we observed statistically significantly larger AUC only for M-CSF, compared to $\mathrm{AUC}=0.5$ (borderline of diagnostic usefulness of the test). Our results showed that the diagnostic accuracy of M-CSF in the group of cervical cancer patients were slightly lower than the diagnostic power of M-CSF reported in our breast cancer study [37] and by Mroczko et al. [38] or by Groblewska et al. [18] in the course of colorectal and pancreatic cancer, and higher compared to non-small cell lung cancer [39]. The remainder of the selected cytokines demonstrated a lower diagnostic power in patients with cervical cancer than in patients with malignant tumors of other locations [18, 38, 40].

Spearman rank correlation was used in the dependence analyses between the investigated cytokines and SCC-Ag. The plasma level of HGFs did not follow the levels of the established gynecological tumor marker. However, we reported earlier a positive correlation between M-CSF and SCC-Ag, which may have resulted from a different composition of the cervical cancer group [25].

In summary, this is the first complete investigation comparing the diagnostic value of the measurement of selected hematopoietic cytokines and the commonly used tumor marker in patients with carcinoma of the uterine cervix. M-CSF appears not to be cancer selective, and alone it is not sufficient for the diagnosis of cervical cancer, although it showed the biggest diagnostic power from all the tested factors. These results suggest a potential role for M-CSF as a tumor marker for the early diagnosis of both cervical intraepithelial neoplasia and cervical cancer, especially in combination with SCC-Ag.

\section{Acknowledgements}

This work was financed by a Grant for Scientific Research (nr N N407 530738) in the years 2010-2013 from the Polish Ministry of Science and Higher Education.

\section{References}

1. Parkin DM, Bray F, Ferlay J, Pisani P. Global center statistics, 2002. CA Cancer J Clin. 2005;55:74-108.

2. Catellsauge X, Diaz M, de Sajnose S et al. Worldwide human papillomavirus etiology of cervical adenocarcinoma and its cofactors: implications for screening and prevention. $J$ Natl Cancer Inst. 2006;98:303-315.

3. Averette HE, Janicek MF. Cervical cancer: prevention, diagnosis and therapeutics. CA Cancer J Clin. 2001;51:92-114.

4. De Lande J, Davelaar EM, Mensdorff-Pouilly S et al. SCC-Ag, lymph node metastases and sentinel node procedure in early stage squamous cell cervical cancer. Gynecol Oncol. 2009;112: $119-125$.

5. Miyasaka N. Introduction: Cytokine receptors and their classification. Nippon Rinsho. 2005;63:Suppl:S5-9.

6. Chao K, Chang C, Yen M, Wang P, Ng H. Granulocyte colony-stimulating factor on the growth of human ovarian carcinoma cells in vitro. Gynecol Obstet Invest. 1999;48:280-284.

7. Baiocchi G, Kavanagh JJ, Talpaz M, Wharton JT, Gutterman JU, Kurzrock R. Expression of the macrophage colony-stimulating factor and its receptor in gynecologic malignancies. Cancer. 1991;67:990-996.

8. Inoue M, Kyo S, Fujita M, Enomoto T, Kondoh G. Coexpression of the c-kit receptor and the stem cell factor in gynecological tumors. Cancer Res. 1994;54:3049-3053.

9. Hammes LS, Tekmal RR, Naud P et al. Up-regulation of VEGF, c-fms and COX-2 expression correlates with severity of cervical cancer precursor (CIN) lesions and invasive disease. Gynecol Oncol. 2008;110:445-451.

10. Kirma N, Hammes LS, Liu YG et al. Elevated expression of the oncogene c-fms and its ligand, the macrophage colony-stimulating factor-1, in cervical cancer and the role of transforming growth factor-beta1 in inducing c-fms expression. Cancer Res. 2007;67:1918-1926.

11. Nasu K, Inoue C, Takai N, Kashima K, Miyakawa I. Squamous cell carcinoma of the cervix producing granulocyte colony-stimulating factor. Obstet Gynecol. 2004;104:1086-1088.

12. Kyo S, Kanaya T, Takakura M, Inoue M. A case of cervical cancer with aggressive tumor growth: possible autocrine growth stimulation by G-CSF and IL-6. Gynecol Oncol. 2000; 78:383-387.

13. Ahn HJ, Park YH, Chang YH et al. A case of uterine cervical cancer presenting with granulocytosis. Korean J Intern Med. 2005;20:247-250.

14. Watanabe A, Wachi T, Omi H et al. Granulocyte colony-stimulating factor-producing small-cell carcinoma of the uterine cervix: report of a case. Diagn Cytopathol. 2000;23:269-274.

15. Ohwada M, Wada T, Saga Y et al. C-kit overexpression in neuroendocrine small cell carcinoma of the uterine cervix. Eur J Gynaecol Oncol. 2006;27:53-55.

16. Haverkos HW. Multifactorial etiology of cervical cancer: a hypothesis. Med Gen Med. 2005;30:57.

17. Bahar B, Cayci B, Coskun U, Buyukberber S, Benekli M, Yildiz R. Granulocyte colony stimulating factor (G-CSF) and macrophage colony stimulating factor (M-CSF) as potential tumor markers in non small cell lung cancer diagnosis. Asian Pacific J Cancer Prev. 2010;11:709-712.

18. Groblewska M, Mroczko B, Wereszczyńska-Siemiątkowska U, Myśliwiec P, Kędra B, Szmitkowski M. Serum levels of granulocyte colony-stimulating factor (G-CSF) and macrophage colony-stimulating factor (M-CSF) in pancreatic cancer patients. Clin Chem Lab Med. 2007;45:30-34.

19. Yokoama T, Hyodo M, Hosoya Y et al. Aggressive G-CSF-producing gastric cancer complicated by lung and brain 
abscesses, mimicking metastates. Gastric Cancer. 2005;8: 198-201.

20. Shizmu N, Miyatake R, Esa A. Squamous cell carcinoma of bladder producing granulocyte colony-stimulating factor (G-CSF): a case report. Acta Urol Japonica. 2005;51:121-124.

21. Skates SJ, Horick N, Yu Y et al. Preoperative sensitivity and specificity for early-stage ovarian cancer when combining cancer antigen CA-125II, CA 15-3, CA 72-4, and macrophage colony-stimulating factor using mixtures of multivariate normal distributions. J Clin Oncol. 2004;22:4059-4066.

22. Ławicki S, Będkowska GE, Gacuta-Szumarska E, Czygier M, Szmitkowski M. The plasma levels and diagnostics utility of stem cell factor and macrophage colony stimulating factor in endometrial cancer patients. Przegl Lek. 2007;64:987-990 .

23. Punnonen R, Teisala K, Kuoppala T, Bennett B, Punnonen J. Cytokine production profiles in the peritoneal fluids of patients with malignanat or benign gynecologic tumors. Cancer. 1998;83:788-796.

24. Connor JP. Aggressive carcinosarcoma of the uterine cervix associated with high levels of granulocyte colony stimulating factor: case report and laboratory correlates. Gynecol Oncol. 2006;103:349-353.

25. Ławicki S, Będkowska GE, Gacuta-Szumarska E, Knapp P, Szmitkowski M. The plasma levels and diagnostics utility of stem cell factor (SCF) and macrophage-colony stimulating factor (M-CSF) in cervical cancer patients. Pol Merkur Lek. 2008;25:38-42.

26. Davelaar EM, Lande J, Mensdorff-Pouilly S, Blankenstein MA, Verheijen RHM, Kenemans P. A combination of serum tumor markers identifies high-risk patients with early-stage squamous cervical cancer. Tumor Biol. 2008;29:9-17.

27. Kotowicz B, Fuksiewicz M, Kowalska M, Jońska-Gmyrek J, Bidziński M, Kamińska J. The value of tumor marker and cytokine analysis for the assessment of regional lymph node status in cervical cancer patients. Int J Gynecol Cancer. 2008; 18:1279-1284.

28. Pras E, Willemse PH, Canrinus AA et al. Serum squamous cell carcinoma antigen and CYFRA 21-1in cervical cancer treatment. Int J Radiat Oncol Biol Phys. 2002;52:23-32.

29. Strauss HG, Laban C, Lautenschlager C, Buchmann J, Schneider I, Koelbl H. SCC antigen in the serum as an independent prognostic factor in operable squamous cell carcinoma of the cervix. Eur J Cancer. 2002;38:1987-1991.
30. Rocha-Zavaleta L, Huitron C, Caceres-Cortes JR et al. Interleukin-2 (IL-2) receptor-betagamma signaling is activated by c-Kit in the absence of IL-2, or by exogenous IL-2 via JAK3/STAT5 in human papillomavirus-associated cervical cancer. Cell Signal. 2004;16:1239-1247.

31. Wang HL, Lu DW. Overexpression of c-kit protein is an infrequent event in small cell carcinomas of the uterine cervix. Mod Pathol. 2004;17:732-738.

32. Rony AA, Horowitz IR, Tekmal RR. Serum levels of macrophage colony-stimulating factor- 1 in cervical human papillomavirus infection and intraepithelial neoplasia. $\mathrm{Am} \mathrm{JOb-}$ stet Gynecol. 1999;180:28-32.

33. Zijlmans HJMA, Fleuren GJ, Baelde HJ, Eilers PHC, Kenter GG, Gorter A. Role of tumor-derived proinflammatory cytokines GM-CSF, TNF- $\alpha$, and IL-12 in the migration and differentiation of antigen-presenting cells in cervical carcinoma. Cancer. 2007;109:556-565.

34. Czygier M, Ławicki S, Szmitkowski M, Mroczko B. Plasma granulocyte-macrophage colony stimulating factor (GM-CSF) and activity of of enzymes in granulocytes of breast cancer patients. Przegl Lek. 2006;63:654-657.

35. Hazelbag S, Fleuren GJ, Baelde JJ, Schuuring E, Kenter GG, Gorter A. Cytokine profile of cervical cancer cells. Gynecol Oncol. 2001;83:235-243.

36. Lawicki S, Czygier M, Gacuta-Szumarska E, Knapp P, Szmitkowski M. The plasma levels and diagnostics utility of granulocyte-colony stimulating factor (G-CSF) and macrophage-colony stimulating factor (M-CSF) in ovarian cancer patients. Pol Merkur Lek. 2006;125:465-468.

37. Ławicki S, Szmitkowski M, Wojtukiewicz M. The pretreatment plasma level and diagnostics utility of M-CSF in benign breast and breast cancer patients. Clin Chim Acta. 2006;371: $112-116$.

38. Mroczko B, Szmitkowski M, Okulczyk B. Hematopoietic growth factors in colorectal cancer patients. Clin Chem Lab Med. 2003;41:646-651.

39. Kamińska J, Kowalska M, Kotowicz B et al. Pretreatment serum levels of cytokines and cytokine receptors in patients with non-small cell lung cancer, and correlations with clinicopathological features and prognosis. Oncology. 2006;70:115-125.

40. Mroczko B, Groblewska M, Wereszczyńska-Siemiątkowska U, Kędra B, Konopko M, Szmitkowski M. The diagnostics value of G-CSF measurement in the sera of colorectal cancer and adenoma patients. Clin Chim Acta. 2006;371:143-147. 